\title{
Tension Hemothorax in
}

\section{Decompensated Alcoholic Cirrhosis}

\author{
Brenden Hoff ${ }^{1}$, \\ Teuta Marsic MD2, \\ Nicole Siparsky MD³
}


- Obstructive shock: final stage of mechanical impedance of venous return to the heart.

- Heralded by cardiovascular collapse, or tension physiology

- Commonly caused by tension pneumothorax (TP).

- Hemothorax rarely cause of tension physiology 
- $38 \mathrm{Y} / \mathrm{O} \mathrm{M} \mathrm{w/} \mathrm{alcoholic} \mathrm{cirrhosis} \mathrm{(MELD} \mathrm{18).}$ Jaundiced, left shoulder/chest pain, SOB

- CT identified chest wall hematoma

- Underwent embolization of two left thryocervical arterial trunk branches

- Hematoma advanced into left thorax despite embolization 
- Arrived to our hospital tachypneic, absent left lung sounds, tracheal deviation to right

- MELD score now 38 ( $\mathrm{Na} 127 \mathrm{mmol} / \mathrm{L}, \mathrm{Cr} 1.9 \mathrm{mg} / \mathrm{dL}$, total bilirubin $72 \mathrm{mg} / \mathrm{dL}$, AST $85 \mathrm{U} / \mathrm{L}$, ALT $27 \mathrm{U} / \mathrm{L}$, INR 2.0, lactic acid $1.7 \mathrm{mmol} / \mathrm{L}$ )

- Initial CXR

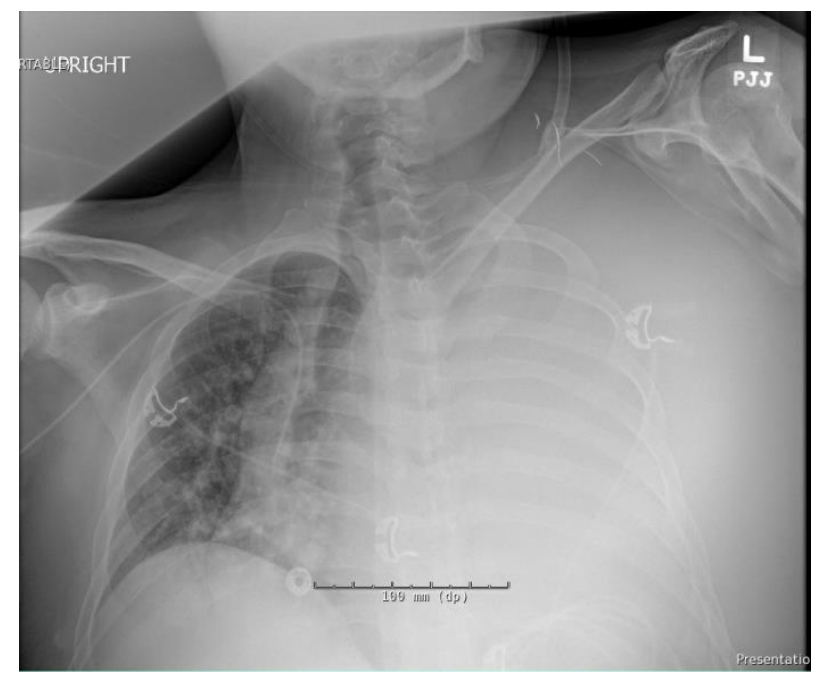




\section{Case Presentation}

- Initial CT Scan

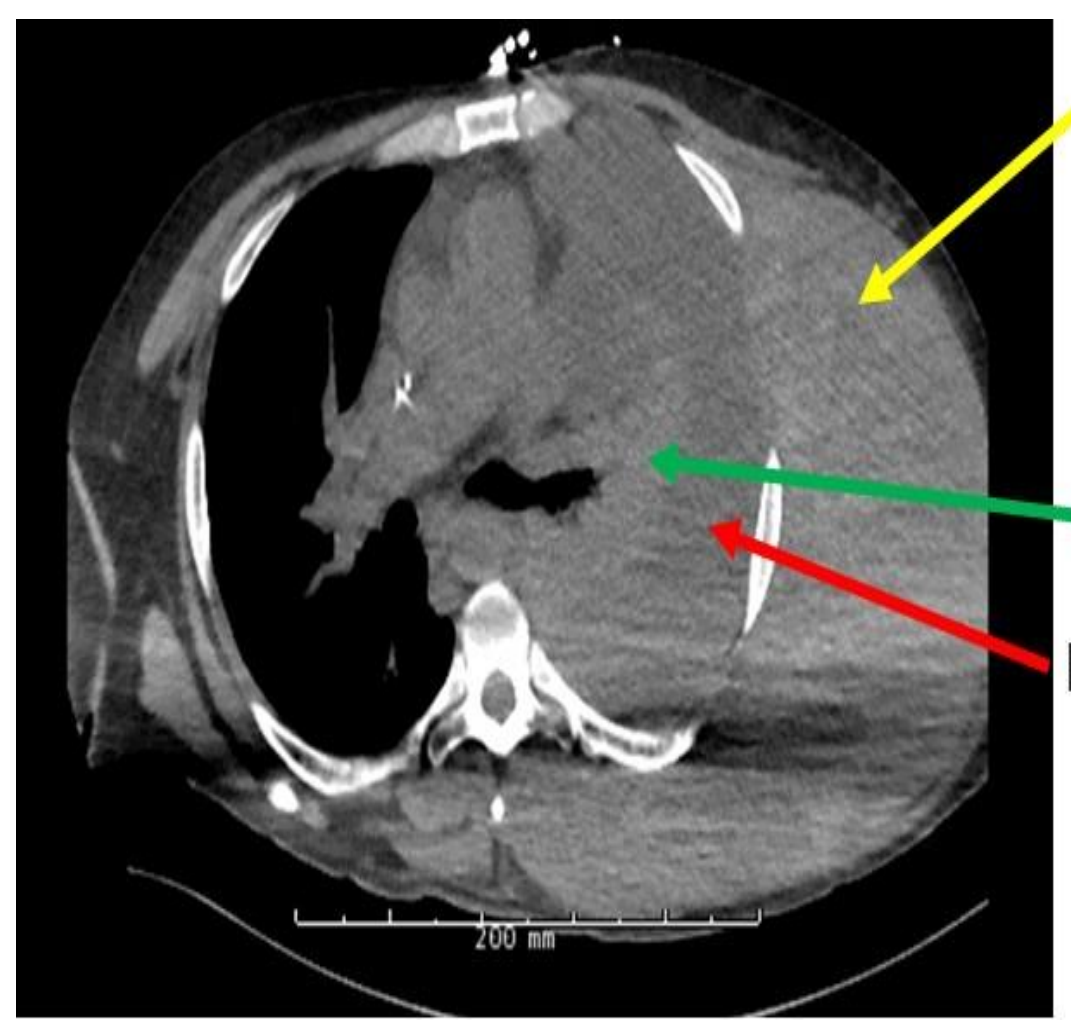

Chestwall hematoma

Deviated trachea

Collapsed

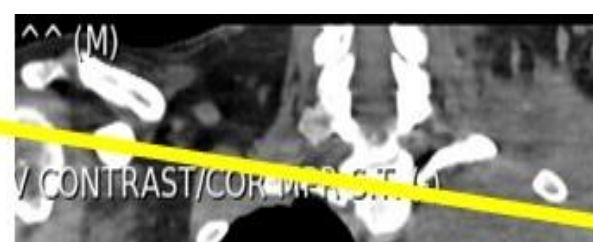

$+2$

- lung

Hemothorax

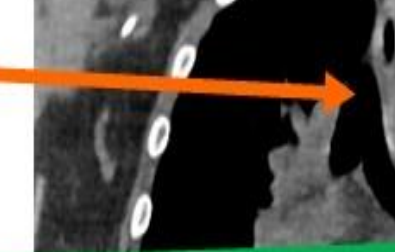

10

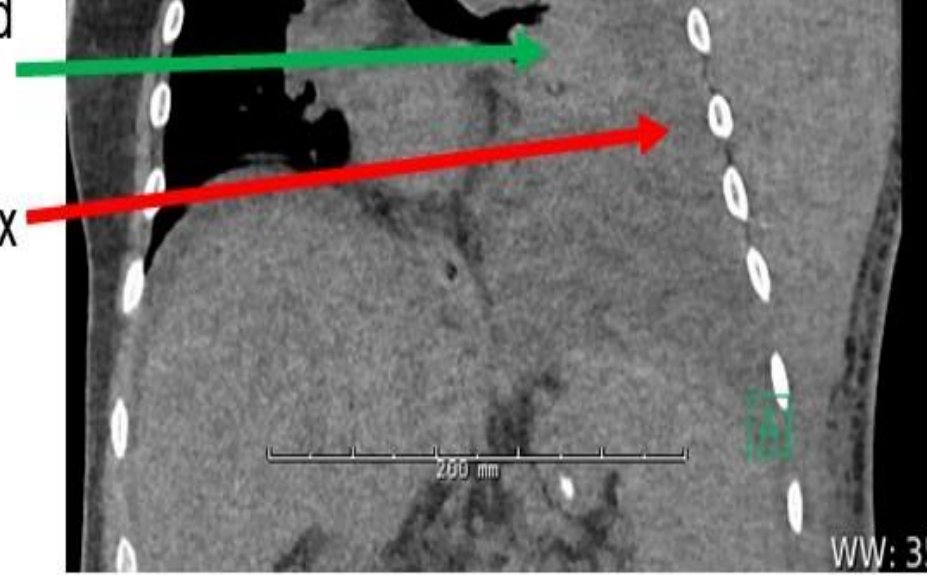




\title{
Case Presentation
}

- Diagnoses:

- decompensated cirrhosis with worsening

- coagulopathy

- acute renal failure

- acute renal failure
- Dialysis
- Mechanical ventilation

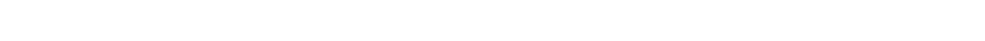

\author{
encephalopathy
}

(

\section{- Treatments
- Balanced transfusion \\ - Treatments
- Balanced transfusion}

$\bullet$ 
- Developed spontaneous retroperitoneal hematoma causing hemorrhagic shock

- Worsening obstructive shock due to abdominal compartment syndrome and tension hemothorax

- Repeat CT

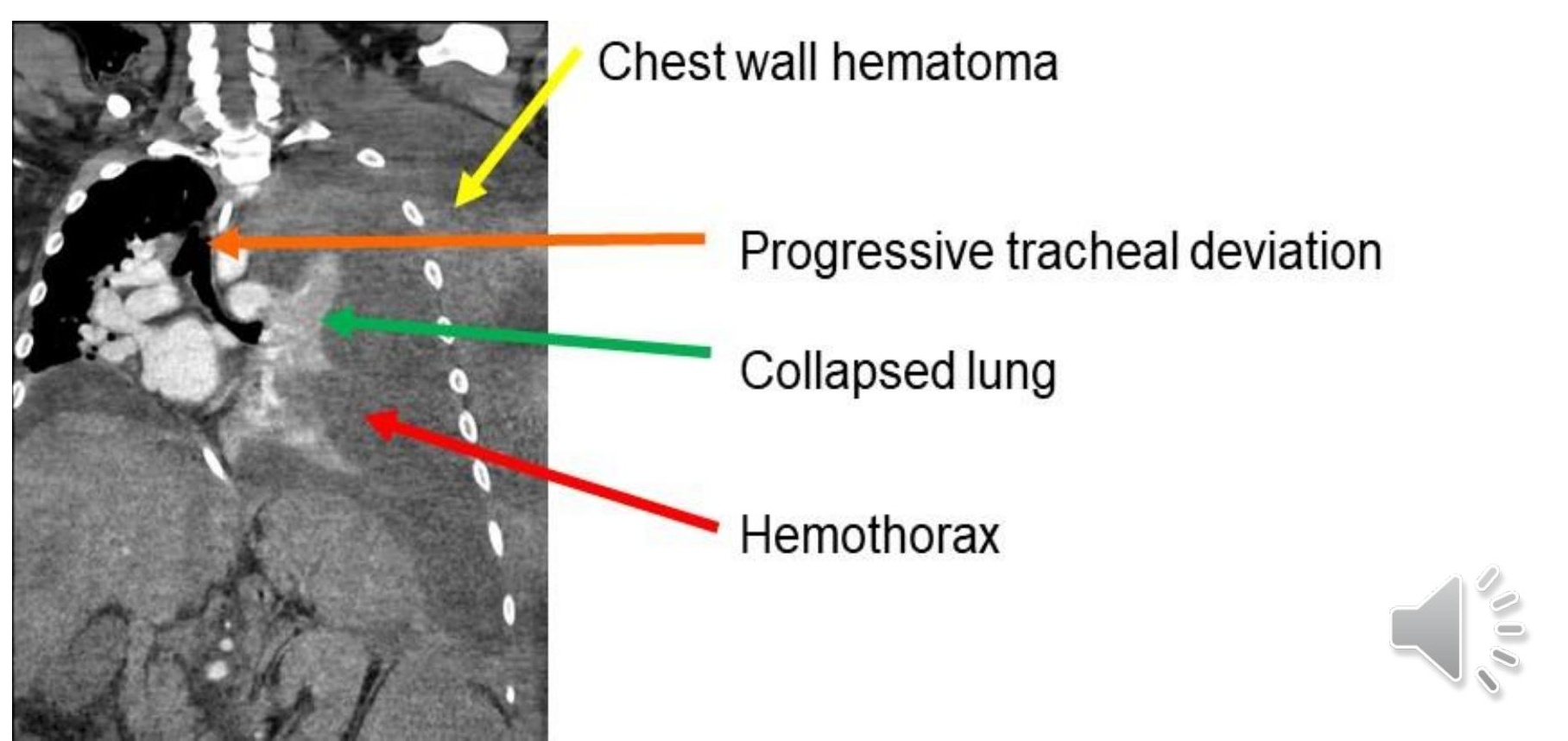


- Became bradycardic and suffered PEA arrest despite appropriate transfusional resuscitation

- Hemoglobin 8g/dL, platelets $75 \mathrm{~K} / \mathrm{uL}$, INR 1.6, lactic acid $9 \mathrm{mmol} / \mathrm{L}$

- Transiently responded to chest compressions and code drugs

- Bedside echocardiogram: diffuse hypokinesis, adequate heart filling, dilated inferior vena cava 
- Identify tension physiology early

- Commonly from pneumothorax

- Hemothorax should be in differential

- Decompensated cirrhotics with hemothorax difficult to manage

- Coagulopathy and poor surgical candidacy

- Hemothorax can progress to tension physiology if bleeding source unable to be controlled

- May ultimately need liver transplant or will succumb to tension hemothorax 


\section{References}

1. Dippel W.F., Doty D.B., Ehrenhaft J.L. Tension hemothoraxdue to patent ductus arteriosus. NEJM 1973; 288:353-354.

2. Shiloni E, Meretyk S, Weis s Y. Tension haemothorax: an unu sual complication of central vein catheterization. Injury 1985 May; 16(6): 385-6.

3. Kawamura M, Takahashi Y, Gouko T, Hoshino R, Ookubo T, Hanaoka N, Satou M. A case of penetrating chest injury with tension hemothoraxby gunshot. Kyobu Geka 1994 Jul; 47(7): 577-9.

4. MartinezF.J., Villanueva A.G., Pickering R, BeckerF.S., Smith D.R. Spontaneous hemothorax. Report of 6 cases and review of the literature. Medicine 1992; 71(6): 354-368.

5. Tan J, AlvarezJ.M. Tension haemothorax: an uncommon life-threatening complication. ANZ J Surg 2002 Jan; $72(1)$ : $72-4$.

6. Yajima K, Urabe N, As ai K, Kageyama Y. Tension hemopneumothoraxcomplicated by severe hepatic and renaldis order; report of a case. Kyobu Geka. 2004 Sep;57(10):993-5.

7. Ka-Ho L, Hon-Da A, Kin-Kong L. Tension hemothorax: a dreaded complication of percutaneous liver biopsy. Clin Gastroenterol He patol. 2010 Jul; 8(7): A36.

8. Morita Y, Ichimura H, KikuchiS, Ozawa Y, Inoue K, Uchida A, Kikuchi K, Shiig ai M, Shiotani S. Spontaneous tension hemothoraxdue to rupture of a solitary fibrous tumor of the posterior mediastinum. Kyobu Geka. 2013 Oct; 66(11): 1006-9.

9. Inafuku K, Imoto K, Kano K, Amano S, Cho T, Minami T, Uchida K, Masuda M. Tension hemothoraxaccompanying rupture of the descending aortic ane ury sm. Kyobu Geka. 2014 Nov; 67(12): 1056-9.

10. Yoshida C, Yamamoto Y, Sas a S, Matsuoka H, Hokimoto N, Yamai H, Ohnishi K, Tanida N, Fujishima N, HamaguchiN, Ichikawa Y, Tano K. Tension Hemothorax As sociated with Spontaneous Rupture of the Thoracic Aorta; Report of a Case. Kyobu Geka. 2015 Aug; 68(9): 789-92.

11. Fourdrain A, Lafitte S, Iquille J, DeDominicis F, Berna P. Delayed-onset tension hemothorax following blunt trauma and the rupture of a previously undiagnosed aberrant right subclavian artery. J Thorac Dis. 2017 Mar; 9(3): E245-E248.

12. Nakayama T, Uehara H, Osawa M, Tanahashi Y, Kondo H, Kawamura M. Atraumatic tension hemothorax as sociated with ruptured aneury smof intercostal artery azygous fistula: a case report. Gen Thorac Cardiovasc Surg. 2018 Sep; 66(9): 552-555.

13. Magnone S, Gotti R, Giulii Capponi M, Paderno N, Maraglino C, Cadei M, Mario C, Lucianetti A. Tension hemothoraxdue to iatrogenic subclavian artery perforation: Hybrid management of a very rare complication. Int J Surg Case Rep. 2019; 60: 323-326.

14. Bidad R, Hall C, Blohm E. Fatal Tension Hemothorax Combined With Exanguination: A Rare Complication of Neurofibromatosis. Clin Pract Cases Emerg Med. 2019 Sep 18; 3(4): 364-368. 